\title{
O absenteísmo da equipe de enfermagem de um hospital universitário público
}

\section{The absenteeism of the team of nursing of a public university hospital}

Larissa Gutierrez de Carvalho Silva ${ }^{1}$, Adeline Aparecida Queiroz Buss² ${ }^{2}$ Maria do Carmo Lourenço Haddad ${ }^{1}$, Marli Terezinha Oliveira Vannuchi ${ }^{1}$

1. Docente do Departamento de Enfermagem da Universidade Estadual de Londrina (UEL), PR, Brasil. 2. Supervisora de enfermagem do pronto atendimento do Hospital Santa Paula (SP), PR, Brasil.

\begin{abstract}
Resumo
Introdução: A identificação do absenteísmo da enfermagem no contexto hospitalar constitui-se em um importante indicador de análise para a gestão de pessoas e para a qualificação e segurança da assistência aos clientes. Objetivo: Analisar o absenteísmo dos trabalhadores da equipe de enfermagem de um hospital público universitário. Metodologia: Pesquisa de abordagem quantitativa, tendo como população os atestados dos profissionais da equipe de enfermagem do ano de 2009. Os dados foram coletados com um instrumento que continha informações sobre o perfil sóciodemográfico e ocupacional, a causa e o período da ausência dos profissionais. A análise descritiva foi realizada, agrupando-se em distribuição de frequências e gráficos. Resultados: 0 perfil dos trabalhadores que apresentaram atestados são mulheres casadas, com idade entre 41 a 50 anos, auxiliares, vínculo empregatício público, tempo de trabalho de 11 a 15 anos, lotados no centro cirúrgico em período integral. 0 número de atestados foi 3.149, 72,46\% por justificativas médicas, o que resultou na ausência de 71,6 trabalhadores de enfermagem por semana. Conclusão: 0 número elevado de atestados médicos pode sugerir um processo de trabalho que deve ser reavaliado sob o enfoque da preservação da saúde dentro do ambiente de trabalho e da subjetividade do cuidar de si e do próximo.
\end{abstract}

Palavras-chave: Absenteísmo. Administração de Recursos Humanos em Hospitais. Equipe de Enfermagem. Serviços de Enfermagem.

\begin{abstract}
Introduction: The identification of nursing absenteeism in hospitals constitutes an important indicator for the analysis of the management of people and the qualification and safety of care of sick people. Objective: To analyze the absenteeism of workers of the nursing staff of a public university hospital. Methodology: A quantitative research approach, based upon the attestations of the nursing team professionals of 2009 was carried out. The data were collected by means of an instrument that contained information on sociodemographic and occupational profiles, as well as the causes and length of the absence of the professionals. A descriptive analysis was performed, grouping in frequency distribution and graphs. Results: The profile of the workers who submitted certificates to justify their absenteeism incclude married women, aged 41 to 50 years old, auxiliaries, public employees, working time from 11 to 15 years, allocated in the operating room on a full-time basis. The amount of certificates was $3.149,72.46 \%$ of which claiming medical reasons, resulting in the lack of 71.6 nursing staff per week. Conclusion: The high number of medical certificates may suggest a work process that should be re-evaluated aiming at the preservation of health in the workplace and the objective of taking care of themselves as well as the others.
\end{abstract}

Keywords: Absenteeism. Personnel Administration Hospital. Nursing Team. Nursing Services.

INTRODUÇÃO

Os diversos significados atribuídos ao trabalho sofrem influências do contexto histórico, econômico, social e são construídos pela coletividade. A partir dos anos de 1960, iniciaram-se as reflexões acerca das relações estabelecidas entre o homem, sua forma de viver e seu trabalho, que são considerados fatores determinantes para seu adoecimento e subsidiam o modelo da determinação social do processo de saúde-doença do indivíduo ${ }^{1}$.

Considerando o trabalho como uma atividade inerente ao homem e reconhecendo a relação com o adoecimento do indivíduo, pode-se ter uma exposição maior aos riscos em um ambiente hospitalar, por ser um local que expõe os trabalhadores aos produtos químicos, aos materiais e patologias infectocontagiosos e às radiações ionizantes ${ }^{2}$.

Dentro deste contexto hospitalar, encontra-se o trabalhador da área de enfermagem exercendo atividades de forma contínua em finais de semana, feriados e/ou em datas comemorativas, com jornadas de trabalho semanais, na maioria das vezes, superiores a quarenta horas, o que contribui para seu desgaste físico. Além disso, ele vivencia um processo de trabalho com altos níveis de estresse advindos do convívio com o sofrimento e a dor do outro, levando-o também ao desgaste psicoemocional ${ }^{3}$.

Há de se considerar ainda que esses trabalhadores, em termos quantitativos, representam uma parcela significativa dos recursos humanos dentro da instituição hospitalar e são a principal força de trabalho na assistência direta prestada ao paciente. Sua ausência no trabalho, denominada de absenteísmo, representa um custo financeiro relevante à instituição e também influencia, de forma negativa, a organização do trabalho em equipe, acarretando sobrecarga aos demais membros ${ }^{4}$.

Considera-se o absenteísmo como o tempo de trabalho perdido

Correspondência: Larissa Gutierrez de Carvalho Silva. Av. Robert Koch, 60, Vila Operária. CEP: 86038-440 - Londrina, PR - Brasil. E-mail: larissagutierrez@ yahoo.com.br

Conflito de interesse: Não há conflito de interesse por parte de qualquer um dos autores.

Recebido em: 28 Jan 2016; Revisado em: 13 Abr 2016; 25 Abr 2016; Aceito em: 10 Maio 2016 
pelos trabalhadores quando eles deixam de comparecer ao ambiente de trabalho. Estas ausências são classificadas como absenteísmo previsto e não previsto. O previsto refere-se às folgas e férias, e o não previsto, aos dias relativos às licenças médicas, licença maternidade, licença por acidente de trabalho, faltas justificadas e injustificadas, outras licenças (nojo, gala, paternidade) e suspensões ${ }^{5}$.

O conhecimento do absenteísmo dentro da realidade dos serviços hospitalares constitui-se em um importante indicador de análise aos gerentes de enfermagem tanto na qualificação e a segurança da assistência aos clientes, como também na administração das equipes de trabalho na área de enfermagem. Este estudo tem como objetivo analisar o absenteísmo em trabalhadores da equipe de enfermagem de um hospital público universitário.

\section{MÉTODO}

Foi realizado um estudo transversal, descritivo, de abordagem quantitativa, utilizando dados secundários. O local do estudo foi o maior hospital público do norte do Paraná, que integra o Sistema Único de Saúde (SUS) para o atendimento em diversas especialidades médicas e cirúrgicas, sendo referência em serviço de alta complexidade. Esta instituição conta com 310 leitos, sendo 124 destinados à internação da clínica médicocirúrgica.

Os dados foram coletados por meio de um instrumento que continha informações sobre o perfil sóciodemográfico e ocupacional, a(s) causa (s) e o(os) período(s) de ausência dos profissionais. Foram incluídos na pesquisa 3.148 atestados para justificar ausência(s) de trabalhadores da equipe de enfermagem, apresentados ao setor de recursos humanos da instituição, no período de 1 o de janeiro a 31 de dezembro de 2009. Os dados demográficos dos trabalhadores foram obtidos por meio do sistema informatizado, Gestão de Pessoas - Versão Delfhi ${ }^{7}$.

Após a coleta, os dados foram ordenados em planilhas eletrônicas e analisados no Programa Microsoft Excel 2007, com a utilização de métodos descritivos, agrupando-se em distribuição de freqüências e gráficos.

A coleta de dados foi realizada após aprovação do Comitê de Ética em Pesquisa envolvendo seres humanos da Universidade Estadual de Londrina-PR, com parecer $n$ ㅇ 268/2010 e CAAE $n$ 으 0093.0.268.000-10.

\section{RESULTADOS}

Em 2009, foram apresentados ao setor de recursos humanos do hospital 3.148 atestados da equipe de enfermagem, com uma média de 262,3 atestados ao mês. Quando se converteu o número de atestados em horas perdidas de trabalho, resultou em 137.478 horas ao ano e uma média de 11.456 horas que deveriam ter sido trabalhadas no mês. Considerando que a carga horária semanal mais prevalente na instituição era de 40 horas e que a média semanal de horas não trabalhadas foi de 2.864 , houve a ausência de 71,6 trabalhadores por semana na instituição pesquisada.

Os trabalhadores eram em sua maioria do sexo feminino $(88,9 \%)$, casados $(55,3 \%)$ e com idade entre 41 e 50 anos (45,3\%). Em relação ao perfil profissional, o maior número de atestados atribuiu-se aos auxiliares de enfermagem $(65,4 \%)$; que possuem vínculo empregatício estadual $(96,7 \%)$; tempo de trabalho na instituição entre 11 e 20 anos (57,1\%); lotados na unidade de centro cirúrgico $(10,0 \%)$ e que realizam suas atividades em período integral (29,8\%) (Tabela 1$)$.

Tabela 1. Distribuição do número de atestados em relação às variáveis sóciodemográficas e ocupacionais. Londrina, 2009.

\begin{tabular}{|c|c|c|}
\hline Variáveis & $\mathbf{n}$ & $\%$ \\
\hline \multicolumn{3}{|l|}{ Sexo } \\
\hline Feminino & 2799 & 88,9 \\
\hline Masculino & 349 & 11,1 \\
\hline \multicolumn{3}{|l|}{ Idade (anos) } \\
\hline $21-30$ & 73 & 2,3 \\
\hline $31-40$ & 884 & 28,1 \\
\hline $41-50$ & 1426 & 45,3 \\
\hline $51-60$ & 646 & 20,5 \\
\hline $61-70$ & 119 & 3,5 \\
\hline \multicolumn{3}{|l|}{ Estado civil } \\
\hline Casado & 1742 & 55,3 \\
\hline Outros & 1406 & 44,7 \\
\hline \multicolumn{3}{|l|}{ Vínculo empregatício } \\
\hline $\mathrm{CLT}^{*}$ & 103 & 3,3 \\
\hline Etatutário & 3045 & 96,7 \\
\hline \multicolumn{3}{|c|}{ Tempo de admissão (anos) } \\
\hline$\leq 1$ & 259 & 8,2 \\
\hline $2-5$ & 351 & 11,1 \\
\hline $6-10$ & 415 & 13,2 \\
\hline $11-15$ & 929 & 29,5 \\
\hline $16-20$ & 870 & 27,6 \\
\hline $21-25$ & 138 & 4,4 \\
\hline $26-30$ & 73 & 2,3 \\
\hline Maior 30 & 113 & 3,6 \\
\hline \multicolumn{3}{|l|}{ Local de trabalho } \\
\hline Centro cirúrgico & 316 & 10,0 \\
\hline Médico Cir. Nasculina & 246 & 7,8 \\
\hline Pronto Socorro & 227 & 7,2 \\
\hline Central de Material & 221 & 7,0 \\
\hline UTI** adulto & 18 & 6,0 \\
\hline
\end{tabular}

J. Health Biol Sci. 2014; 2(4): 88-94 


\begin{tabular}{lrr}
\hline Variáveis & $\mathbf{n}$ & \% \\
\hline Outros & 1755 & 55,7 \\
Turno & & \\
Integral (8 horas) & 938 & 29,8 \\
Matutino & 768 & 24,4 \\
Noturno & 618 & 19,6 \\
Vespertino & 824 & 26,2 \\
Função & & \\
Auxiliar & 2060 & 65,4 \\
Enfermeiro & 421 & 13,4 \\
Técnico & 667 & 21,2 \\
\hline
\end{tabular}

* Consolidação das Leis Trabalhistas

** Unidade de Terapia Intensiva

O centro cirúrgico apresentou 316 atestados, perfazendo uma média mensal de 26,3 atestados, seguido pela unidade médicocirúrgica masculina, com 246 atestados (7,8\%), pronto socorro, com 227 atestados (7,2\%), central de material, $221(7,0 \%)$ e unidade de terapia intensiva adulto, 188 (6,0\%) (Tabela 1).

Em relação ao tempo de admissão, a tabela 1 ainda evidencia que os trabalhadores com menos de 1 ano tiveram mais ausência ao trabalho $(8,2 \%)$ do que os profissionais com mais de 21 anos de ingresso na instituição $(4,4 \%)$ e aqueles com mais de 26 anos de trabalho no hospital (2,3\%).

Nas unidades de centro cirúrgico, pronto socorro e central de material, há predomínio de atestados entre os trabalhadores com idade entre 41 a 50 anos, diferentemente da unidade médico-cirúrgica masculina em que predomina a faixa etária de 31 a 40 anos.

Observa-se na tabela 2 que o principal tipo de atestado apresentado pela equipe de enfermagem foi a licença médica, representando $72,4 \%$, seguida pelos atestados como acompanhantes em consultas médicas (14,2\%).

De acordo com a tabela 3, a maioria dos atestados teve duração de 1 a 6 horas (49,3\%), e a categoria de auxiliar de enfermagem apresentou a maior quantidade de horas de afastamento (2.060 horas). Se for feito um somatório das horas de afastamento do técnico e do auxiliar de enfermagem, constata-se que os profissionais de nível médio da equipe de enfermagem tiveram 2.727 horas, que equivale a $86,6 \%$ das horas de ausência ao trabalho.

Na tabela 4 , evidencia-se o cruzamento do número de atestados pelas variáveis sóciodemográficas e ocupacional, segundo a categoria profissional.

Tabela 2. Distribuição dos tipos de absenteísmo não previstos por categoria profissional. Londrina 2009.

\begin{tabular}{|c|c|c|c|c|c|c|c|c|}
\hline \multirow[t]{2}{*}{ Tipos de absenteísmo não previsto } & \multicolumn{2}{|c|}{ Enfermeiro } & \multicolumn{2}{|c|}{$\begin{array}{c}\text { Técnico de } \\
\text { enfermagem }\end{array}$} & \multicolumn{2}{|c|}{$\begin{array}{c}\text { Auxiliar de } \\
\text { enfermagem }\end{array}$} & \multicolumn{2}{|c|}{ Total } \\
\hline & $\mathbf{n}$ & $\%$ & $\mathbf{n}$ & $\%$ & $\mathbf{n}$ & $\%$ & $\mathbf{n}$ & $\%$ \\
\hline Atestado médico & 288 & 9,1 & 465 & 14,7 & 1.528 & 48,5 & 2.281 & 72,4 \\
\hline Atestado médico de acompanhante & 69 & 2,2 & 97 & 3,1 & 280 & 8,9 & 446 & 14,2 \\
\hline Atestado por acidente de trabalho & & 0,0 & 1 & 0,1 & 8 & 0,2 & 9 & 0,3 \\
\hline $\begin{array}{l}\text { Participação de cursos, congressos, } \\
\text { eventos científicos }\end{array}$ & 42 & 1,3 & 7 & 0,2 & 193 & 1,6 & 100 & 3,2 \\
\hline Outros & 22 & 0,7 & 97 & 3,1 & 51 & 6,2 & 312 & 9,9 \\
\hline Total & 421 & 13,3 & 667 & 21,1 & 2.060 & 65,4 & 3.148 & 100 \\
\hline
\end{tabular}

Tabela 3. Distribuição de atestados pela duração em horas de afastamento e por categoria profissional. Londrina, 2009.

\begin{tabular}{|c|c|c|c|c|c|c|c|c|}
\hline \multirow{2}{*}{$\begin{array}{c}\text { Duração em } \\
\text { horas }\end{array}$} & \multicolumn{2}{|c|}{ Enfermeiro } & \multicolumn{2}{|c|}{ Técnico de enfermagem } & \multicolumn{2}{|c|}{ Auxiliar de enfermagem } & \multicolumn{2}{|c|}{ Total } \\
\hline & $\mathbf{n}$ & $\%$ & $\mathbf{n}$ & $\%$ & $\mathbf{n}$ & $\%$ & $\mathbf{n}$ & $\%$ \\
\hline $1-6 h$ & 200 & 47,5 & 387 & 58,0 & 965 & 47,0 & 1.552 & 49,3 \\
\hline $7-12 h$ & 75 & 17,8 & 108 & 16,0 & 493 & 24,0 & 676 & 21,5 \\
\hline $13-24 h$ & 52 & 12,3 & 40 & 6,0 & 158 & 8,0 & 250 & 7,9 \\
\hline Outros & 94 & 22,4 & 132 & 20,0 & 444 & 21,0 & 670 & 21,3 \\
\hline Total & 421 & 100,0 & 667 & 100,0 & 2.060 & 100,0 & 3.148 & 100,0 \\
\hline
\end{tabular}

Fonte: Autores 
910 absenteísmo da enfermagem de um hospital

Tabela 4. Distribuição do número de atestados segundo variáveis sóciodemográficas e ocupacional, por categoria profissional, Londrina, 2009.

\begin{tabular}{|c|c|c|c|c|c|c|c|c|c|c|c|c|}
\hline \multirow[t]{3}{*}{ Variáveis } & \multicolumn{4}{|c|}{ Enfermeiro } & \multicolumn{4}{|c|}{ Técnico em Enfermagem } & \multicolumn{4}{|c|}{ Auxiliar de Enfermagem } \\
\hline & \multicolumn{2}{|c|}{$\begin{array}{l}\text { Atestado } \\
\text { Médico }\end{array}$} & \multicolumn{2}{|c|}{ Outros } & \multicolumn{2}{|c|}{$\begin{array}{l}\text { Atestado } \\
\text { médico }\end{array}$} & \multicolumn{2}{|c|}{ Outros } & \multicolumn{2}{|c|}{$\begin{array}{l}\text { Atestado } \\
\text { Médico }\end{array}$} & \multicolumn{2}{|c|}{ Outros } \\
\hline & $\mathbf{n}$ & $\%$ & $\mathbf{n}$ & $\%$ & $\mathbf{n}$ & $\%$ & $\mathbf{n}$ & $\%$ & $\mathbf{n}$ & $\%$ & $\mathbf{n}$ & $\%$ \\
\hline \multicolumn{13}{|l|}{ Sexo } \\
\hline Femino & 269 & 93,0 & 113 & 85,0 & 405 & 87,1 & 177 & 87,6 & 1.361 & 89,1 & 474 & 89,1 \\
\hline Masculino & 19 & 7,0 & 20 & 15,0 & 60 & 12,9 & 25 & 12,4 & 167 & 10,9 & 58 & 10,9 \\
\hline \multicolumn{13}{|l|}{ Idade (anos) } \\
\hline $31-40$ & 82 & 28,5 & 47 & 35,3 & 224 & 48,2 & 112 & 55,5 & 296 & 19,4 & 123 & 23,1 \\
\hline $41-50$ & 139 & 48,3 & 65 & 48,9 & 143 & 30,7 & 63 & 31,2 & 731 & 47,8 & 285 & 53,6 \\
\hline Outros & 67 & 23,3 & 21 & 15,8 & 98 & 21,1 & 27 & 13,4 & 501 & 32,8 & 124 & 23,3 \\
\hline \multicolumn{13}{|l|}{ Estado civil } \\
\hline Casado & 146 & 50,7 & 69 & 51,9 & 311 & 66,9 & 134 & 66,3 & 785 & 51,4 & 297 & 55,8 \\
\hline Outros & 142 & 49,3 & 64 & 48,1 & 154 & 33,1 & 68 & 33,7 & 743 & 48,6 & 235 & 44,2 \\
\hline Unidades & 15 & 5,2 & 4 & 3,0 & 75 & 16,1 & 29 & 14,4 & 78 & 5,1 & 26 & 4,9 \\
\hline Pronto Socorro & 15 & 5,2 & 4 & 3,0 & 75 & 16,1 & 29 & 14,4 & 78 & 5,1 & 26 & 4,9 \\
\hline Centro Cirúrgico & 11 & 3,8 & 11 & 8,2 & 54 & 11,6 & 21 & 10,4 & 169 & 11,1 & 44 & 8,3 \\
\hline Central de Material & 17 & 5,9 & 2 & 1,5 & 31 & 6,7 & 10 & 5,0 & 117 & 7,7 & 50 & 9,4 \\
\hline $\begin{array}{l}\text { Médico- Cirúrgica } \\
\text { Masculina }\end{array}$ & 11 & 3,8 & 18 & 13,5 & 71 & 15,3 & 8 & 5,0 & 107 & 7 & 31 & 5,8 \\
\hline Secretaria de Enfermagem & 37 & 12,8 & 23 & 17,3 & - & - & - & - & - & - & - & - \\
\hline Ambulatório-HC & 37 & 12,8 & 5 & 3,8 & - & & - & - & 95 & 6,2 & 15 & 2,8 \\
\hline Outros & 160 & 55,6 & 70 & 52,6 & 234 & 50,3 & 134 & 66,3 & 962 & 62,9 & 366 & 68,8 \\
\hline \multicolumn{13}{|l|}{ Vínculo } \\
\hline Estatutário & 270 & 93,7 & 133 & 100,0 & 387 & 83,2 & 195 & 96,5 & 1.528 & 100 & 532 & 100 \\
\hline Temporário & 18 & 6,2 & - & & 78 & 16,8 & 7 & 3,5 & - & - & - & - \\
\hline \multicolumn{13}{|l|}{ Turno } \\
\hline Vespertino & 56 & 19,4 & 23 & 17,3 & 269 & 57,8 & 125 & 61,9 & 261 & 17,1 & 90 & 16,9 \\
\hline Integral & 155 & 53,8 & 74 & 55,6 & 15 & 3,2 & 11 & 5,4 & 538 & 35,2 & 145 & 27,3 \\
\hline Outros & 77 & 26,7 & 36 & 27,1 & 181 & 38,9 & 66 & 32,7 & 729 & 47,7 & 297 & 55,8 \\
\hline \multicolumn{13}{|l|}{ Tempo de admissão (anos) } \\
\hline$\leq 1$ & 44 & 15,4 & 13 & 9,8 & 148 & 31,8 & 54 & 26,7 & - & & - & \\
\hline 2 a 5 & - & & 11 & 8,3 & 203 & 43,7 & 118 & 58,4 & - & & - & \\
\hline 11 a 15 & 71 & 24,6 & 31 & 23,3 & 40 & 8,6 & 13 & 6,4 & 591 & 38,7 & 183 & 34,4 \\
\hline 16 a 20 & 62 & 21,5 & 33 & 24,8 & 55 & 11,8 & 15 & 7,4 & 505 & 33,1 & 200 & 37,6 \\
\hline Outros & 111 & 38,5 & 45 & 33,8 & 19 & 4,1 & 2 & 1,0 & 432 & 28,2 & 149 & 28,0 \\
\hline
\end{tabular}

*HC: Hospital das Clínicas

Fonte: Autores 
Analisando as categorias profissionais na tabela 4, os achados revelam que o maior número de atestados ocorreu entre os auxiliares de enfermagem; as profissionais do sexo feminino; casadas; com faixa etária entre 41 a 50 anos; trabalho em período integral; vinculo empregatício estadual; tempo de trabalho entre 11 a 15 anos na instituição e lotadas na unidade de centro cirúrgico. A maioria dos atestados foi por licença médica com afastamento do trabalho.

Entre os enfermeiros, os resultados foram semelhantes aos encontrados entre os auxiliares de enfermagem, exceto pela unidade de lotação, que no caso dos enfermeiros, teve uma maior frequência entre aqueles da diretoria de enfermagem (Tabela 4).

Na categoria dos técnicos de enfermagem, a faixa etária que mais se ausentou foi entre 31 e 40 anos, com tempo de trabalho na instituição entre dois a cinco anos, com horário de trabalho no turno vespertino e lotados na unidade de pronto socorro (Tabela 4).

\section{DISCUSSÃO}

O absenteísmo da população estudada apresenta-se elevado e a preocupação acerca desses valores se dá pelo tempo de trabalho que deveria ter sido desenvolvido pelos profissionais de enfermagem, e também pela redução inesperada da força de trabalho já programada e contabilizada no contexto de cuidado ao paciente, trazendo um impacto negativo sobre a organização do trabalho e a qualidade de assistência prestada ${ }^{6}$.

Em instituições hospitalares, os profissionais estão sujeitos a desenvolver suas atividades de trabalho com um número insuficiente de pessoal devido às ausências não previstas de trabalhadores, o que gera sobrecarga sobre a equipe de enfermagem e agrava o desgaste emocional e físico presentes neste cotidiano de trabalho. Essa realidade produz um efeito cascata entre esses profissionais, pois o trabalhador submetido à sobrecarga de trabalho em decorrência da ausência de seu colega apresenta um risco maior aos agravos à saúde do trabalhador e, consequentemente, o risco de ausentar-se do trabalho torna-se ainda maior ${ }^{4}$.

Historicamente, a enfermagem apresenta-se como uma profissão caracterizada por um número significativo de mulheres constituído por sua principal força de trabalho. Isso deve ser considerado quando vários estudos acerca do absenteísmo da equipe de enfermagem revelam que as mulheres são responsáveis por parte significativa do valor das ausências não previstas $^{6,7,8}$.

Nota-se, neste estudo, uma maioria de mulheres entre a faixa etária de 30 a 49 anos, consideradas como mulheres adultas jovens. É importante ressaltar que, nessa faixa etária, são diversas as responsabilidades dessas mulheres, tanto no desenvolvimento do papel social de esposa e mãe no âmbito particular, quanto nas atividades profissionais, sendo isso um grande fator de sobrecarga tanto emocional quanto física ${ }^{9,10}$.
Estudo retrospectivo sobre o absenteísmo revelou que a maioria era mulheres, com idade entre 31 e 40 anos, embora a segunda maior causa de afastamento sejam transtornos mentais e comportamentais. Para essas mulheres, a falta de tempo para desenvolver atividades físicas, alimentar-se adequadamente, ter horas de sono e repouso adequados aumenta o risco de agravos à sua saúde, impedindo que elas atuem no sentido da prevenção de doenças e agravos à sua saúde?.

Além desses fatores, essa população ainda pode estar sujeita aos sintomas do período do climatério, os quais podem interferir em sua concepção de qualidade de vida e, consequentemente, influenciar sua atuação no ambiente laboral ${ }^{11}$.

A alta frequência de atestados entre os trabalhadores com vínculo empregatício estadual encontrado neste estudo corrobora resultados de ooutras pesquisas sobre a prevalência do absenteísmo e os dias de afastamentos entre servidores públicos. Esses autores sugerem que essas ausências podem estar relacionadas ao sentimento de maior segurança e estabilidade entre aqueles vinculados ao Estado em que ainda se tolera um número maior de ausências do servidor público quando comparado ao setor privado ${ }^{7,8,12}$.

Um levantamento sobre os fatores associados ao absenteísmo por doença autorreferida em trabalhadores de enfermagem de três hospitais públicos no município do Rio de Janeiro sugere chances três vezes $(O R=3,12$ ) mais elevadas entre os servidores públicos de haver ausência ao trabalho. Os autores também enfatizam a possível influência do tempo de admissão na instituição com a alta prevalência de atestados entre os trabalhadores, que pode estar vinculada a baixas condições laborais e, consequente, redução da motivação profissional ${ }^{12}$.

Em contrapartida, um estudo sobre o absenteísmo em unidade intensiva identificou número reduzido de dias perdidos de trabalho apresentados pelos estatutários, o que nos sugere maior comprometimento com a instituição devido à remuneração salarial diferenciada ${ }^{13}$.

A autopercepção do estatutário em uma instituição pública apresenta-se associada às questões como a estabilidade e, principalmente, a tolerância com determinados padrões de comportamento e segurança7, 8,12; entretanto, essa percepção pode trazer prejuízos à gerência das instituições caso não sejam adotadas medidas eficazes de controle como a avaliação de desempenho profissional.

Vale ressaltar que o quantitativo do absenteísmo desses trabalhadores pode ser uma variável de extrema importância tanto para uma avaliação rigorosa sobre o desempenho profissional, quanto para o processo gerencial visto que as horas de trabalho perdidas demandam um acréscimo nos custos do serviço em decorrência da necessidade de substituição dessa mão de obra ausente ${ }^{14}$.

Confirmando os dados encontrados neste estudo a respeito da freqüência de atestados apresentados pelos enfermeiros, 
técnicos e auxiliares, outras investigações corroboram o fato de que são os auxiliares os maiores responsáveis pelo número de ausências $7,8,12,15$.

Este achado pode estar associado à responsabilidade dos auxiliares de enfermagem na execução de atividades de natureza ergonômica e repetitiva caracterizadas pela sobrecarga física de ter seu trabalho menos valorizado social e financeiramente e frequentemente exercer dupla jornada de trabalho $0^{9,16}$.

Em contrapartida, no cotidiano do enfermeiro, há o predomínio das atividades gerenciais que pode desencadear aumento do sentimento de responsabilidade e seu comprometimento com a equipe. Além disso, este profissional desenvolve a gestão do serviço de enfermagem, o que Ihe proporciona menor tempo de exposição aos riscos se comparado aos auxiliares e técnicos, o que pode ser considerado um dos fatores que reduzam suas ausências no trabalho ${ }^{8}$.

Ressalva-se que, em uma unidade de terapia intensiva, na qual o enfermeiro executa algumas atividades, assim como o profissional de nível técnico, e se expõe aos mesmos riscos, observa-se ainda maior assiduidade quando comparado ao profissional técnico ${ }^{13}$.

Outra influência sobre o quantitativo de atestados entre a população estudada foi o local onde esse trabalhador desenvolve suas atividades profissionais, sendo as unidades como o Centro Cirúrgico e o Pronto Socorro as que se destacaram, assim como em outros estudos sobre absenteísmo $0^{9,10,11}$.

Alguns autores consideram que os ambientes e as situações de urgência/emergência, como aquelas vivenciadas diuturnamente em unidades como Pronto Socorro e Centro Cirúrgico, são considerados desencadeadores de desgaste psíquico que vitimam os trabalhadores de enfermagem, possibilitando a ocorrência de angústia, estresse e distúrbios emocionais que podem resultar em eventos de acidentes e afastamento do trabalho ${ }^{4,7}$.

Ao analisarmos os tipos de atestados apresentados, a licença médica de uma a 12 horas de afastamento e licença médica para o acompanhamento de familiares foram os tipos mais frequentes entre todas as categorias profissionais. Corroboram o nosso estudo diversas pesquisas sobre o absenteísmo entre a equipe de enfermagem, sendo a maioria das ausências não previstas decorrentes de licenças médicas, sejam elas por períodos curtos ou prolongados $7,8,10,12,15,17$.

Sabe-se que nem todos os atestados por licenças médicas significam $o$ adoecimento deste profissional, entretanto devemos conhecer a psicodinâmica deste processo de trabalho a fim de reconhecer o significado e as consequências dessa ausência no trabalho.

Deve ser considerado que estes profissionais são submetidos a diversos riscos no ambiente laboral, como biológicos, químicos, físicos ergonômicos ${ }^{7,16}$, e que há um desgaste emocional causado por um contato excessivo com os outros seres humanos. Em contraponto a todos esses fatores também existe uma valorização excessiva dos sinais e sintomas em que a proximidade aos profissionais médicos facilita o acolhimento de suas queixas.

Outro ponto a ser investigado são os dados sobre o afastamento do profissional por decorrência de acidente de trabalho ausência decorrente de algum acidente sofrido durante a jornada ou percurso do trabalho - que tem uma baixa frequência neste estudo, sugerindo uma notificação inadequada ou até uma subnotificação desses dados considerados tão importantes ${ }^{16}$.

De acordo com estudo realizado na mesma instituição sobre os acidentes de trabalho ocorridos no ambiente hospitalar, 54,7\% foram sofridos pelos trabalhadores de enfermagem. Este dado representa a existência de uma exposição real dos trabalhadores a acidentes no exercício da atividade laboral ${ }^{18}$.

De forma estratégica, têm sido desenvolvidos estudos aprofundados sobre o processo de trabalho em instituições hospitalares uma vez que as melhorias nos processos de produção poderão ter reflexos significativos, em termos de qualidade de atendimento à população, e relacionados à diminuição de custos para o sistema público de saúde ${ }^{19}$.

No mesmo sentido, o controle de gastos é um instrumento de trabalho fundamental para a otimização das operações das instituições, sobretudo dos hospitais públicos, os quais são financiados pelas próprias ações e serviços executados, visto que precisam apresentar relatórios de produtividade que evidenciem os procedimentos realizados. Dessa maneira, o absenteísmo dos trabalhadores pode significar queda de produtividade e menos recursos a serem aplicados ${ }^{17}$.

Esta economia pode ser não apenas direta, com redução de custos operacionais, mas também indireta, na medida em que poderá haver reflexos positivos que propiciam a promoção da saúde, a prevenção de doenças, diminuem os agravos à saúde, bem como proporcionam uma melhor qualidade de vida a esses profissionais.

\section{CONCLUSÃO}

A partir da análise dos 3148 atestadas da equipe de enfermagem no ano de 2009, verificou-se que houve uma considerável quantidade de horas de afastamento (137.478 horas no ano) na instituição, o que resultou na ausência de 71,6 trabalhadores de enfermagem por semana.

Em relação ao perfil sóciodemográfico e ocupacional, os trabalhadores eram, em sua maioria, do sexo feminino $(88,9 \%)$, casados $(55,3 \%)$, com idade entre 41 e 50 anos $(45,3 \%)$, da categoria "auxiliar de enfermagem" (65,4\%), com vínculo empregatício estadual (96,7\%), tempo de trabalho na instituição entre 11 e 20 anos (57,1\%), lotados na unidade de centro cirúrgico $(10,0 \%)$ e que realizam suas atividades em período integral $(29,8 \%)$. O principal tipo de atestado foi a 
licença médica, com duração de 1 a 6 horas.

Evidencia-se com este estudo a necessidade de traçar a identificação do perfil de adoecimento dos trabalhadores de enfermagem por meio do Serviço Especializado em Engenharia de Segurança e Medicina do Trabalho subsidiado pelo Programa de Controle Médico de Saúde Ocupacional (PCMSO) sob o enfoque de prevenção, rastreamento e diagnóstico precoce dos agravos à saúde, de natureza subclínica.

Além do perfil de adoecimento desse trabalhador, pode haver intervenções em conjunto com a Diretoria de Enfermagem e
Educação Continuada da Instituição a fim de preservar a saúde e a integridade física e mental dos trabalhadores, como a realização de programas que resgatem o sentimento de equipe e interdependência, que visem à preservação da saúde dentro do ambiente de trabalho e da subjetividade do cuidar de si e do próximo.

Acredita-se que estes elementos contribuiriam para a redução do adoecimento desse profissional e que resultaria em um aumento na assiduidade e melhoria na qualidade e segurança da assistência prestada.

\section{REFERÊNCIAS}

1. Martins JT, Robazzi MLCC, Bobroff MCC. Prazer e sofrimento no trabalho da equipe de enfermagem: reflexão à luz da psicodinâmica Dejouriana. Rev Esc Enferm USP. 2010 Dez; 44(4):1107-11. doi: http://dx.doi.org/10.1590/S008062342010000400036 .

2. Silva ARS, Souza KRF, Silva ICP, Silva JG, Oliveira JMS. Meio Ambiente hospitalar e o risco ocupacional da equipe de enfermagem: uma revisão integrativa. Cadernos de Graduação - Ciências Biológicas e da Saúde Facipe. 2013Ago; 1(1):11-20, 2013.

3. Silva GT, Cunha CRT, Costa ALRC, Maruyama SAT. Experiência de adoecimento e licença médica: o caso de uma técnica de enfermagem. REME rev. min. enferm. 2013Jan-Mar; 17(1): 207-215.

4. Martinato MCNB, Svero DF, Marchand EAA, Siqueira HCH. Absenteísmo na enfermagem: uma revisão integrativa. Rev Gaucha Enferm. 2010 Mar; 31(1):160-6. http://dx.doi.org/10.1590/S1983-14472010000100022.

5. Chiavenato I. Gestão de pessoas: o novo papel dos recursos humanos nas organizações. 4 ed. Barueri: Manole; 2014.

6. Cucolo DF, Perroca MG. Absenteeism in the nursing team in surgical-clinical units of a philanthropic hospital. Acta paul. enferm. 2008; 21(3): 454-459. doi: http://dx.doi.org/10.1590/S0103-21002008000300012.

7. Mininel VA, Felli VEA, Silva EJ, Torri Z, Abreu AP, Branco MTA. Workloads, strain processes and sickness absenteeism in nursing. Rev. Latino-Am. Enfermagem. 2013 Nov-Dez; 21(6): 1290-1297. doi: http://dx.doi.org/10.1590/01041169.2992.2366.

8. Sancinett TR, Soares AVN, Lima AFC, Santos NC, Melleiro MM, Fugulin FMT, Gaidzinski RR. Taxa de absenteísmo da equipe de enfermagem como indicador de gestão de pessoas. Rev. esc. enferm. USP. 2011 Ago; 45(4): 1007-1012. doi: http://dx.doi.org/10.1590/S0080-62342011000400031.

9. Santana LL, Miranda FMD, Karino ME, Baptista PCP, Felli VEA, Sarquis LMM. Cargas e desgastes de trabalho vivenciados entre trabalhadores de saúde em um hospital de ensino. Rev. Gaúcha Enferm. 2013 Mar; 34(1): 64-70. doi: http:// dx.doi.org/10.1590/S1983-14472013000100008.

10. Ferreira EV, Amorim MJDM, Lemos RMC, Ferreira NS, Silva FO, Loureano JR Filho. Absenteísmo dos trabalhadores de enfermagem em um hospital universitário do Estado de Pernambuco. Rev Rene. 2011 Out-Dez; 12(4): 742-9.

11. Márquez Membrive J, Granero-Molina J, Sova Salmeron MJS, FernándezSola C, Rodrigues López CMR, Parrón Carreño T. Quality of life in perimenopausal women working in the health and educational system. Rev. Latino-Am. Enfermagem. 2011Nov-Dez; 19(6):1314-1321. PubMed PMID: 22249664.

12. Ferreira RCA, Griep RH, Fonsea MJM, Rotenberg L. Abordagem multifatorial do absenteísmo por doença em trabalhadores de enfermagem. Rev. Saúde Pública. 2012 Abr: 46(2): 259-268. doi: http://dx.doi.org/10.1590/S003489102012005000018.

13. Inoue KC, Matsuda LM, Silva DMP, Uchimura PTT, Mathias TA. The absenteeism-disease of a nursing team in an intensive care unit. Rev. Bras. Enferm. 2008 Mar-Apr; 61(2): 209-214. PubMed PMID: 18572841.

14. Umann J, Guido LA, Leal KP, Freitas EO. Absenteísmo na equipe de enfermagem no contexto hospitalar. Ciênc. cuid. saúde. 2011; 10(1):184-190. doi: 10.4025/cienccuidsaude.v10i1.11867

15. Primo GMG, Pinheiro TMM, Sakurai E. Absenteísmo por doença em trabalhadores de uma organização hospitalar pública e universitária. Rev med Minas Gerais. 2010 Abr-Jun; 20(2, Supl.2): S47-58.

16. Giomo DB, Freitas FCT, Alves LA, Robazzi MLCC. Occupational accidents, occupational risks and absenteeism among hospital nursing workers. Rev. enferm. UERJ. 2009 Jan-Mar; 17(1):24-9.

17. Junkes MB, Pesso VF. Financial Expense Incurred by Medical Leaves of Health Professionals in Rondonia Public Hospitals, Brazil. Rev. Latino-Am. Enfermagem. 2010 Maio-Jun; 18(3): 406-412. doi: http://dx.doi.org/10.1590/ S0104-11692010000300016.

18. Sêcco IAO, Robazzi MLCC, Shimizu DS, Rúbio MMS. Typical occupational accidents involving workers at a university hospital in southern Brazil: epidemiology and prevention. Rev. Latino-Am. Enfermagem. 2008 Set-Out; 16(5): 824-831. doi: http://dx.doi.org/10.1590/S0104-11692008000500005.

19. Lancman S, Gonçalves RMA, Mângia EF. Organização do trabalho, conflitos e agressões em uma emergência hospitalar na cidade de São Paulo, Brasil. Rev. Ter. Ocup. Univ. 2012 Set-Dez; 23(3):199-207. doi: http://dx.doi.org/10.11606/ issn.2238-6149.v23i3p199-207. 